\title{
Electroencephalographic Findings after Eslicarbazepine Therapy in Focal Epileptic Syndrome Patients
}

\author{
Gabriel Salazar*, Marta Fragoso, Javier Codas \\ Department of Neurology, Hospital Terrassa CST, Terrassa, Spain \\ Email: *gsalazar@cst.cat
}

How to cite this paper: Salazar, G., Fragoso, M. and Codas, J. (2018) Electroencephalographic Findings after Eslicarbazepine Therapy in Focal Epileptic Syndrome Patients. World Journal of Neuroscience, $\mathbf{8}$, 342-349.

https://doi.org/10.4236/wjns.2018.83027

Received: May 1, 2018

Accepted: July 20, 2018

Published: July 23, 2018

Copyright (@) 2018 by authors and Scientific Research Publishing Inc. This work is licensed under the Creative Commons Attribution International License (CC BY 4.0).

http://creativecommons.org/licenses/by/4.0/

(c) (i) Open Access

\begin{abstract}
The efficacy and the tolerance of the AEDs have been extensibly studied in the past, however the effects of them on the EEG activity of epileptic patients have been scarcely studied. Eslicarbazepine is a third generation blocker-sodium channels AED associated with a high reduction in the rate of partial seizures in epileptic patients. We designed an open label, non control study to determine the effects of Eslicarbazepine on the EEG activity of EP with focal seizures in a 12-week follow up. The EP with focal paroxystical activity enrolled in this study showed a statistical significant reduction in the rate of monthly seizures when Eslicarbazepine $800 \mathrm{mgs}$ was added to a previous AED compared to those patients who only received an increase of the AED in monotherapy, concomitantly, EP treated with ESL added to a previous AED showed a statistically significant reduction in the mean occipital frequency and voltage amplitude in the central and parietal regions in the twelfth-week EEG compared to the control group.
\end{abstract}

\section{Keywords}

EEG, Focal Epilepsy, Anti-Epileptic Drugs

\section{Introduction}

The clinical and molecular effects of antiepileptic drugs (AEDs) have been extensively investigated in the past, however much less is known about their effects on Electroencephalography [1] [2] [3].

Some AEDs as carbamazepine and lamotrigine (both, channel blockers) and valproic acid have been related to electroencefalography changes in epileptic patients. Slowing of the posterior (occipital) frequency has been related to the im- 
provement in the rate of seizures in epileptic patients (EP) after some AEDs therapy [4]. Even though, there is little information about the effects of new generation AEDs on the EEG activity in epileptic patients, some AEDs, such as levetiracetam are associated with acceleration of background EEG frequencies and improved cognitive function. Other AEDs have been related with changes in the paroxysmal EEG activity, however these findings couldn't be reproduced in further studies. Most of the past studies about AEDs have stressed the focus on the efficacy and tolerance of the AEDs leaving behind in a second place the pharmacological mechanism of action of these drugs. Interestingly, some authors have pointed out the usefulness of EEG nor only in the diagnosis but in the prognosis of the EP patients as a diagnostic tool in order to follow up the epileptic focus nevertheless there are not scientific evidences that can confirm this statement. On the other hand, there are basic and neurophysiological studies that give some clues about possible action mechanism of some AEDs, for example, change in frequency power band could predict improvement in several cognitive domains across Levetiracetam therapy [5]. Lacosamide modulates interictal spiking and high-frequency oscillations in a model of mesial temporal lobe epilepsy [6]. Eslicarbazepine treatment prevented acute latrunculin A-induced seizures, as well as chronic seizures and all EEG chronic signs of paroxysmal activity, supporting a possible anti-epileptogenic effect of Eslicarbazepine in mice [7] [8]. There are few studies related to the EEG activity pre and post AED in EP patients to our knowledge. We designed a prospective, open label, parallel controlled follow up of EP with a focal syndrome to study the EEG characteristics pre and post Eslicarbazepine therapy using QEEG analysis.

\section{Patients and Methods}

Epileptic patients with clinical and EEG criteria of focal epileptic syndrome were enrolled in this 12-week, prospective, open label, parallel control-study.

\subsection{Inclusion and Exclusion Criteria}

Focal EP of both sexes, from 18 to 80 years old with a poor control of seizures, with only one anti-epileptic drug, and who needed an adjustment of the AED therapy according to the real clinical practice, in order to control seizures frequency were included in this study. Patients should have a focal-paroxysmal EEG activity at the screening visit and clinical criteria of partial simple or complex seizures at the beginning of the study. Patients and/or caregivers should understand adequately how to fill up the seizures diary. Patients with pseudo-seizures even having real seizures were excluded of the study as well as EP patients under therapy with drugs that can potentially can modify the EEG activity finally EP patients with non control systemic disease also were not included in this study.

\subsection{Methods}

Patients were randomized subsequently into two groups (A and B), Group A pa- 
tients received Eslicarbazepine (ESC) as an add-therapy to another anti-epileptic drug. $400 \mathrm{mgs}$ once a day the first week then after, $800 \mathrm{Mgs}$ once a day from the second week until the end of the study (12 weeks), and Group B patients received an AED in monotherapy, but adjusting the therapy through an increase in dose according to the usual clinical practice.

Patients were visited every four weeks: Visit 1 -screening visit and Basal EEG; Visit 2-1 week later, randomization; Visits 3 and 4-clinical control, 4 and 8 weeks later; Visit 5-final EEG and clinical control, 12 weeks after randomization.

Patients were instructed to fill up seizures diary and adverse effects questionnaires, which were asked in all the visits after randomization. All the patients signed a written consent form before being included in the study.

Two EEGs were performed to all the patients of both groups: the first one at the screening visit and the second one at the end of the study. The EEG was a 30 minute registering (Nihon Kohden Co., Japan) with activation maneuvers. The EEG was analysed by visual objective experience and by a software of EEG parameters analyser, in all the EEG regions; 80 ephocs free of artifacts with paroxysmal EEG activity were analysed, and other 80 ephocs without paroxysmal activity. QEEG analysis of bands power: Alfa $(8-13 \mathrm{~Hz})$, theta $(4-8 \mathrm{~Hz})$, Delta (1 - $4 \mathrm{~Hz})$, beta $(14-18 \mathrm{hz})$ and gamma (>18). The QEEG of the Paroxystical activity were analysed separately.

\subsection{Statistical Analysis}

The endpoint assessment measures were the EEG-parameters and the seizures diary changes from baseline to the end of the study. The Statistical analysis was performed comparing the basal data to the twelve-week evaluation data of both groups.

Statistical significance was determined $p<0.05 \mathrm{~T}$ student was the statistical method used and SPSS pack statistical analysis was used.

\section{Results}

$40 \mathrm{EP}$ were screened, 3 of them were excluded because they did not meet an EEG focal paroxysmal activity at the screening visit. Other 5 patients were excluded because they showed no clear partial seizures.

32 patients were included in the study, all of whom were able to finish the 12 -week follow up, they were randomized into two groups (A and B), $15 \mathrm{EP}$ patients in group A, Eslicarbazepine added to another anti-epileptic drug (SCL + $\mathrm{AED}$ ), and $17 \mathrm{EP}$ patients were included in Group B (AED monotherapy).

Patients of Group A showed a mean age of 57 yo (Aged from 36 to $67 \mathrm{SD} \pm 8$ years), 9 women and 6 men. Mean Years of diagnostic of 14 years \pm 3 . Patients of the Group B showed similar demographic characteristics with a mean of age of 42 yo, (20 to $56 \mathrm{SD} \pm 9,11$ years) 11 women and 6 men, years of diagnostic $9 \pm 3$ (Table 1). 
Table 1. Demographic characteristics.

\begin{tabular}{ccccccc}
\hline N = 32 & N & Sex: F/M & Age (mean) Years onset & $\begin{array}{c}\text { Monthly } \\
\text { seizures }\end{array}$ & $\begin{array}{c}\text { Simple/complex } \\
\text { seizures }\end{array}$ \\
\hline $\begin{array}{c}\text { Group A } \\
(\text { ESC + AED) }\end{array}$ & 15 & $9 / 6$ & $57(36-67)$ & $14 \pm 3$ & $3.4 \pm 0.4$ & $6 / 9$ \\
$\begin{array}{c}\text { Group B (AED } \\
\text { monotherapy) }\end{array}$ & 17 & $11 / 6$ & $42(20-56)$ & $9 \pm 3$ & $3.2 \pm 0.2$ & $7 / 8$ \\
\hline
\end{tabular}

With regard to epileptic seizures, patients of Group A (ESC + AED) showed at basal evaluation a mean of monthly seizures of $3.8 \pm 0.4$, posteriorly they showed a mean of monthly seizures of $1.5 \pm 0.2$ in the twelfth week evaluation (average of reduction of $-59.3 \%, p<0.05$ ). Patients of Group B (AED in monotherapy) showed a reduction of monthly seizures from $3.2 \pm 0.2$ at basal evaluation to 2.2 \pm 0.8 seizures in the twelfth week evaluation visit (average of reduction of $-31 \%$, $p<0.05$ ) (Table 2).

With regard to EEG findings in areas of non paroxistic activity, EP of group A showed a reduction in the occipital rate from 12.3 to $8.3 \mathrm{~Hz}(-35 \%, p<0.05)$ and also showed a reduction in the central parietal amplitude from $20 \mathrm{v} \pm 2$ to 10 $\mathrm{v} \pm 1(-50 \%, p<0.05)$ at the end of the follow up, however the rest of the brain regions showed no changes in the neurophysiological parameters.

Patients of Group B showed reduction in the occipital frequency from $10.2 \mathrm{~Hz}$ to $9.2 \mathrm{~Hz}(-9.9 \%, p>0.05)$ at the end of the study. They showed no other relevant changes in the rest of neurophysiological parameters in the different brain areas at the end of the study (Table 3).

In relation to the EEG characteristics in the areas of paroxysmal activity, patients of Group A showed changes in the mean frequency of the paroxysmal activity from $4.3 \mathrm{~Hz}$ to $2.6 \mathrm{~Hz}$. (reduction $30 \%, p<0.05$ ) at the 12 week follow up. They showed no significant changes in the mean of amplitude from 42.4 to 42.3 $\mathrm{V}(p>0.05)$ at the end of the study. There were no changes in relation to the location and the morphology of the focal paroxysmal activity at the end of the study. Patients of Group B showed no significant reduction in the mean of frequency of the Paroxysmal activity from 4.6 to $4.2(p>0.05)$. They showed no changes in the mean of amplitude of the paroxysmal activity at the end of the study (Table 4 \& Table 5).

\section{Discussion}

Both groups of Epileptic patients of this study showed a statistical significant reduction of monthly frequency seizures at the end of the study. However, Patients treated with ESL-in ADD-on therapy to another AED showed a greater reduction in monthly seizures $(-59.3 \%$ monthly seizures) compared to the monotherapy group ( $-33 \%$ monthly seizures). This is not a novel data because it has been published in the past that partial syndrome EP had shown a greater 
Table 2. Follow up of the frequency of monthly seizures in both groups.

\begin{tabular}{ccccc}
\hline & $\begin{array}{c}\text { Basal seizures } \\
\text { (mean) }\end{array}$ & $\begin{array}{c}\text { 12-week } \\
\text { (mean) }\end{array}$ & $\begin{array}{c}\text { \% reduction } \\
\text { seizures }\end{array}$ & $p<0.05$ \\
\hline $\begin{array}{c}\text { Group A (ESC + AED) } \\
\text { Group B (AED } \\
\text { monotherapy) }\end{array}$ & $3.8 \pm 0.4$ & $1.5 \pm 0.2$ & $59.3 \%$ & $p<0.001$ \\
\hline
\end{tabular}

Table 3. EEG findings in the ephocs of free paroxistical activity, basal and at 12-week evaluation in the Esc + AED group.

\begin{tabular}{ccccc}
\hline Esc + AED & Basal & 12-week & \% reduction & $p$ \\
\hline Occipital & & & & \\
Rate & $12.3 \mathrm{~Hz}$ & $8.3 \mathrm{~Hz}$ & -35 & $p<0.001$ \\
Voltage & $20 \mathrm{v} \pm 2$ & $10 \mathrm{v} \pm 1$ & -50 & $p<0.001$ \\
Parietal & & & & \\
Rate & $7.2 \mathrm{~Hz}$ & $6.8 \mathrm{~Hz}$ & -0.6 & $p>0.001$ \\
Voltage & $10 \mathrm{v} \pm 1$ & $10.3 \mathrm{v} \pm 1$ & +0.3 & $p>0.001$ \\
temporal & & & & \\
Rate & $6.3 \mathrm{~Hz}$ & $6.4 \mathrm{~Hz}$ & +0.1 & $p>0.001$ \\
Voltage & $8 \mathrm{v} \pm 0.8$ & $7.8 \pm 0.4$ & -2.5 & $p>0.001$ \\
Frontal & & & & $p>0.001$ \\
Rate & $18 \pm 1$ & $17.3 \pm 0.4$ & -3.8 & $p>0.001$ \\
Voltage & $5 \mathrm{v} \pm 0.4$ & $5 \mathrm{v} \pm 0.4$ & 0 & \\
\hline
\end{tabular}

Table 4. EEG parameters in the epochs free of paroxistical activity, basal and at the 12 -week evaluation in the AED monotherapy group.

\begin{tabular}{ccccc}
\hline AED monotherapy & Basal & 12-week & \% reduction & $p$ \\
\hline Occipital & & & & \\
Rate & $10.2 \mathrm{~Hz}$ & $9.2 \mathrm{~Hz}$ & -0.8 & $p>0.001$ \\
Voltage & $18 \mathrm{v} \pm 3$ & $17 \mathrm{v} \pm 0.7$ & -0.5 & $P>0.001$ \\
Parietal & & & & \\
Rate & $6.2 \mathrm{~Hz}$ & 5.9 & -0.1 & $P>0.001$ \\
Voltage & $12 \mathrm{v} \pm 1$ & $12.5 \pm 1$ & +0.5 & $P>0.001$ \\
temporal & & & & \\
Rate & $5.8 \mathrm{~Hz}$ & $5.6 \mathrm{~Hz}$ & +0.1 & $p>0.001$ \\
Voltage & $6 \mathrm{v} \pm 0.4$ & $7.8 \pm 0.4$ & -2.5 & $p>0.001$ \\
Frontal & & & & \\
Rate & $16.3 \pm 0.7$ & $16.8 \pm 0.4$ & +3 & $p>0.001$ \\
Voltage & $4 \mathrm{v} \pm 0.5$ & $5.2 \pm 0.3$ & +2.5 & $p>0.001$ \\
\hline
\end{tabular}

Table 5. EEG parameter in the epochs of paroxistical activity, basal and at the 12-month evaluation.

\begin{tabular}{cccc}
\hline Paroxysmal activity & Basal & 12-week & $p$ \\
\hline Esc + AED & & & \\
Rate Hz & 4.3 & 2.6 & $p<0.01$ \\
Amp & 42.4 & 42.3 & $p>0.01$ \\
AED monotherapy & & & \\
Rate & 4.6 & 4.8 & $p>0.01$ \\
Amp & 34 & 36 & $p>0.01$ \\
\hline
\end{tabular}


reduction in the monthly seizures when a second AED is added in order to control seizures [9] [10].

In regards to the EEG findings in the epochs of free paroxysmal activity, we observed interesting data in the ESC group which consists in the slowing of the mean posterior frequency from 12.3 to $8.3 \mathrm{~Hz}(-35 \%, p<0.05)$ at the end of this study. This finding is consistent with other studies published before in which had reported that other AEDs decreased the posterior frequency presumably related to the blockage of the sodium/potassium voltage gated channels [11] simultaneously, patients of this group showed a reduction in the centre parietal voltage amplitude from $20 \mathrm{v} \pm 2$ to $10 \mathrm{v} \pm 1(-50 \%, p<0.05)$ at the end of the study, this data has not been reported in other studies in the past to our knowledge, unlike the monotherapy-group which showed a mild and not statistical representative change in the EEG parameters at the end of the study. Even though patients of group B (Monotherapy) showed a reduction in the monthly frequency seizures the decrease in the rate of seizure was greater in ESL group, and we also observed that the greater reduction in the posterior EEG activity is directly related to the decrease in the frequency of monthly seizures; some authors have published that Carbamazepine and Oxcarbazepine $(\mathrm{Na} / \mathrm{K}$ blockers channels) could cause similar EEG findings in epileptic patients [12] [13], but this has not been stated in EP patients treated with Eslicarbazepine to our knowledge.

In regards to the EEG activity in the epochs of paroxystical activity, the patients of ESL group also showed a reduction in the mean frequency of the paroxistical activity decreasing from $4.3 \mathrm{~Hz}$ at the basal EEG to $2.6 \mathrm{~Hz}$ (reduction $30 \%$, $p<0.05$ ) at the end of the study, however there were no changes in the mean voltage amplitude in the epochs of paroxysmal activity (from basal $50 \pm 8$ to the end point $55 \pm 7 p>0.05$ ). Patients of the monotherapy group showed no EEG changes, neither in frequency nor in amplitude in the epochs of paroxysmal activity at the end of the study.

There were no relevant changes in the location and the morphology of the focal paroxystical activity of both groups at the end of the study.

There is some published data about important changes in the EEG parameters in EP after AED therapy as we pointed out above, interestingly AEDs, such as Phenytoin, valproic acid and carbamazepine have been shown to change EEG parameters in EP patients and in models' rats [14]. However there is little published information in relation to EEG charges in EP patients treated with ESL as well as in mice.

ESL treatment prevented acute latrunculin A-induced seizures, as well as chronic seizures and all EEG chronic signs of paroxysmal activity, supporting a possible antiepileptogenic effect in mice.

We have not a clear scientific explanation in regards to the EEG changes in the ESL group, however ESL has shown in mice a greater selectivity of the inactive state of Voltage gate sensitive channel, which is the common feature of the 
rapidly firing neurons, over their resting state as compared to CBZ, OXC [15].

We could hypothesize that this selectivity of interaction of the ESL with the inactive state of the voltage-gated sodium channel (VGSC) could determine greater effects on the EEG of EP patients [16]. ESL in add on therapy to another AED could cause a greater blockage of the $\mathrm{Na}+\mathrm{K}+$ depending channels, which could decrease the posterior EEG frequency and the voltage-amplitude in all the EEG in the free-paroxistic activity epochs, as well as in the epochs where the paroxystical focal activity was observed in the EP patients of this study.

\section{Conclusions}

The EP with focal paroxystical activity of this study showed a statistical significant reduction in the rate of monthly seizures when Eslicarbazepine $800 \mathrm{mgs}$ was added to a previous AED compared to those patients who only received an increase of the AED in monotherapy, concomitantly, EP treated with ESL added to a previous AED showed a statistically significant reduction in the mean occipital frequency and voltage amplitude in the central and parietal regions in the twelfth week EEG compared to the control group.

There was a strong correlation between the reduction in the monthly seizures and the reduction in the EEG electrical activity (occipital frequency and voltage amplitude in central and parietal regions) in the Eslicarbazepine group in the twelfth week evaluation.

We are aware of the limitation of this study (short sample, open study), however this data gives us a clue about possible neurophysiological effects and action mechanism of ESL as AED Voltage-gated sodium channels blocker and also a possible forecast value of EEG in the control of seizures frequency in EP patients treated with Eslicarbazepine, however better designed studies should confirm these results taking into account the outcomes and complications in longer clinical trials.

\section{Consent}

All the patients signed a written consent before being included into the study, this Study was ap-proved by the local committee of ethic.

\section{References}

[1] Brodie, M.J. and Dichter, M.A. (1997) Established Antiepileptic Drugs. Seizure, 6, 159-174. https://doi.org/10.1016/S1059-1311(97)80001-5

[2] Cramer, J.A., Fisher, R., Ben Menachem, E., French, J. and Mattson, R.H. (1999) New Antiepileptic Drugs: Comparison of Key Clinical Trials. Epilepsia, 40, 590-600. https://doi.org/10.1111/j.1528-1157.1999.tb05561.x

[3] Mattson, R.H., Cramer, J.A., Collins, J.F., et al. (1985) Comparison of Carbamazepine, Phenobarbital, Phenytoin, and Primidone in Partial and Secondarily Generalized Tonic-Clonic Seizures. New England Journal of Medicine, 313, 145-151. https://doi.org/10.1056/NEJM198507183130303

[4] Clemens, B., Ménes, A., Piros, P., Bessenyei, M., Altmann, A., Jerney, J., Kollár, K., 
Rosdy, B., Rózsavölgyi, M., Steinecker, K. and Hollódy, K. (2006) Quantitative EEG Effects of Carbamazepine, Oxcarbazepine, Valproate, Lamotrigine, and Possible Clinical Relevance of the Findings. Epilepsy Research, 70, 190-199. https://doi.org/10.1016/j.eplepsyres.2006.05.003

[5] Cho, J.R., Koo, D.L., Joo, E.Y., Yoon, S.M., Ju, E., Lee, J., et al. (2011) Effect of Levetiracetam Monotherapy on Background EEG Activity and Cognition in Drug-Naïve Epilepsy Patients. Clinical Neurophysiology: Official Journal of the International Federation of Clinical Neurophysiology, 123, 883-891.

https://doi.org/10.1016/j.clinph.2011.09.012

[6] Behr, C., Lévesque, M., Ragsdale, D. and Avoli, M. (2015) Lacosamide Modulates Interictal Spiking and High-Frequency Oscillations in a Model of Mesial Temporal Lobe. Epilepsy Research, 115, 8-16. https://doi.org/10.1016/j.eplepsyres.2015.05.006

[7] Sierra-Paredes, G., Loureiro, A., Wright, L.C., Sierra-Marcuño, G. and Soares-da-Silva, P. (2014) Effects of Eslicarbazepine Acetate on Acute and Chronic Latrunculin A-Induced Seizures and Extracellular Amino Acid Levels in the Mouse Hippocampus. BMC Neuroscience, 15, 134. https://doi.org/10.1186/s12868-014-0134-2

[8] Aires, C., Hebeisen, S. and Soares-da-Silva, P. (2012) Effects of Eslicarbazepine, R-Licarbazepine and Oxcarbazepine on Fast and Slow Inactivation of Voltage-Gated Sodium Channels. Epilepsia, 53, 51.

[9] Goldenberg, M.M. (2010) Overview of Drugs Used For Epilepsy and Seizures. P\&T, 35, 392-415.

[10] Konishi, T., Naganuma, Y., Hongou, K., Murakami, M., Yamatani, M. and Okada, T. (1995) Effects of Antiepileptic Drugs on EEG Background Activity in Children with Epilepsy: Initial Phase of Therapy. Clinical Electroencephalography, 26, 113-119. https://doi.org/10.1177/155005949502600209

[11] Guberman, A. (1998) Monotherapy or Polytherapy for Epilepsy? Canadian Journal of Neurological Sciences, 25, S3-S8. https://doi.org/10.1017/S0317167100034892

[12] Salinsky, M.C., Oken, B.S., Storzbach, D. and Dodrill, C.B. (2003) Assessment of CNS Effects of Antiepileptic Drugs by Using Quantitative EEG Measures. Epilepsia, 44, 1042-1050. https://doi.org/10.1046/j.1528-1157.2003.60602.x

[13] Dam, M., Ekberg, R., Løyning, Y., Yngve, W. and Jakobsen, K. (The Scandinavian Oxcarbazepine Study Group) (1989) A Double-Blind Study Comparing Oxcarbazepine and Carbamazepine in Patients with Newly Diagnosed, Previously Untreated Epilepsy. Epilepsy Research, 3, 70-76. https://doi.org/10.1016/0920-1211(89)90070-3

[14] Salinsky, M.C., Binder, L.M., Oken, B.S., Storzbach, D., Aron, C.R. and Dodrill, C.B. (2002) Effects of Gabapentin and Carbamazepine on the EEG and Cognition in Healthy Volunteers. Epilepsia, 43, 4824-4890. https://doi.org/10.1046/j.1528-1157.2002.22501.x

[15] Claassen, J., Hirsch, L.J., Emerson, R.G. and Mayer, S.A. (2002) Treatment of Refractory Status Epilepticus with Pentobarbital, Propofol, or Midazolam: A Systematic Review. Epilepsia, 43, 146-153. https://doi.org/10.1046/j.1528-1157.2002.28501.x

[16] Soares-da-Silva, P., Pires, N., Bonifácio, M.J., Loureiro, A.I., Palma, N. and Wright, L.C. (2015) Eslicarbazepine Acetate for the Treatment of Focal Epilepsy: An Update on Its Proposed Mechanisms of Action. Pharmacology Research \& Perspectives, 3, e00124. https://doi.org/10.1002/prp2.124 